\title{
Tendencias a nivel nacional de la demanda de Profesionales Aeronáuticos Universitarios
}

Iván Vladimir Betancourt

\section{Resumen}

La finalidad de esta investigación es revelar las características que debe tener el perfil del profesional aeronáutico egresado de la primera carrera a nivel superior aeronáutica del ámbito civil en Honduras, para definir en base a este perfil ideal los contenidos curriculares necesarios, la estructura de los espacios de aprendizaje de cada asignatura, las orientaciones aeronáuticas que deben proponerse, la duración de esta carrera y el número de clases y unidades valorativas con las que debe contar. La metodología empleada incluyó una revisión bibliográfica que da cuenta de las tendencias del ambiente aeronáutico internacional tanto en lo operacional como lo educativo, y la consulta hecha a la población general y a grupos claves del desarrollo de la aviación nacional. Los resultados presentados se derivan de los pronósticos mundiales de la necesidad de personal técnico aeronáutico, de los déficits que deberá enfrentar este personal, de la población mundial dependiente de este campo, así como de las preferencias expresadas por los profesionales consultados por medio de una entrevista estructurada. El aporte considerado más importante es la definición del perfil de la carrera aeronáutica a ser impartida a través del Departamento de Ciencias Aeronáuticas de la Facultad de Ciencias Espaciales de la Universidad Nacional Autónoma de Honduras.

Palabras claves: Perfil profesional, educación aeronáutica, ciencias aeronáuticas, tendencias educativas, demanda de profesionales aeronáuticos.

\section{Abstract}

The purpose of this research is to reveal the characteristics required of the graduate profile of the first professional aviation career on civil aviation area in Honduras, to define based on this ideal profile required curriculum content, structure learning spaces of each subject, aeronautical guidelines to be proposed, the duration of this career and the number of classes and credit units must include. The methodology included a literature review that realizes trends in international aviation environment 
both in operational and educational, and consultation made to the general public and key groups in the development of national aviation. The results presented are derived from global forecasts of the need for aeronautical technical personnel, deficits faced by the staff of the world's population dependent on this field as well as the preferences expressed by professionals consulted by means of a structured interview. The contribution considered more important is the definition of aviation career profile being imparted through the Department of Aeronautical Sciences of the Faculty of Spatial Sciences of the National Autonomous University of Honduras.

Keywords: Professional profile, aviation education, aeronautical sciences, educational trends, demand for aviation professionals.

Iván Vladimir Betancourt, (ivan.betancourt@unah.edu.hn), Departamento de Ciencias Aeronáuticas, Facultad de Ciencias Espaciales, Universidad Nacional Autónoma de Honduras - UNAH 


\section{Introducción}

La presente investigación debe ser entendida en el marco del proceso de conformación del campo de la aeronáutica civil en la UNAH. En este momento ya se cuenta con un Departamento de Ciencias Aeronáuticas y se está conformando una Licenciatura que se espera sea la primera en Honduras, construida en una perspectiva civil. En tal sentido se ha asumido como principal referente la esfera de los técnicos y profesionales de la aeronáutica civil.

Los objetivos de la investigación se proponen dar a conocer datos relevantes en tres áreas, la del mercado para profesionales y técnicos aeronáuticos, la de los conocimientos, competencias y valores que deben poseer estos profesionales y la de la demanda de los técnicos y profesionales mencionados. La metodología utilizada se basa principalmente en un examen de la bibliografía disponible y de páginas web relacionadas, así como en una consulta con profesionales conocedores del campo. Se proponen tres resultados principales: 1) algunos datos del mundo aeronáutico que ofrecen una idea general de cómo se encuentra el mercado mundial de la demanda de formación aeronáutica, 2) las preferencias de las empresas de la aeronáutica civil en Honduras al momento de hacer sus contrataciones y 3 ) un perfil del profesional y el técnico aeronáuticos. Al final se proponen conclusiones y recomendaciones con la finalidad de agilizar el proceso de la incorporación de los perfiles elaborados a la oferta académica del Departamento de Ciencias Aeronáuticas.

\section{Método}

El punto de partida de la investigación fueron los aportes del Documento Bases para el establecimiento de las Ciencias Aeronáuticas en la Facultad de Ciencias Espaciales de la UNAH, este documento define el campo aeronáutico en Honduras y sus tendencias atribuyendo un papel central al personal aeronáutica en el desarrollo del campo; se explica con datos de la Autoridad Aeronáutica Hondureña que 752 técnicos del área aeronáutica deben hacer frente a un parque de aeronaves con 114 unidades, 147 aeródromos y 46 empresas aeronáuticas (Pineda, Amaya y Otros, 2012). Otro elemento importante de la revisión bibliográfica fue el estudio realizado por la OACI para proyectar las tendencias del mundo aeronáutico hasta el Año 2030; entre otros aspectos este documento hace una prospectiva de cuáles serán los perfiles profesionales con mayor demanda y cuál será el déficit de profesionales con el que se deberá lidiar (OACl, 2011). Por otra parte se consideran relevantes los aportes de la OIT sobre el entorno aeronáutico actual, el organismo reporta que el transporte aéreo en 2007 generó un valor añadido de 425,000 
millones de Dólares y proveyó de trabajo en forma directa a 5,6 millones de personas en el mundo con un espectro muy variado de perfiles profesionales que incluyó personal de diseño, construcción y mantenimiento de aeronaves, pilotos, personal de cabina, controladores, empleados de servicio al cliente, maleteros y agentes de seguridad entre otros (Organización Internacional del Trabajo, 2013).

Para explorar las preferencias de las empresas del rubro aeronáutico se aplicó una encuesta a profesionales y técnicos activos en la industria y la institucionalidad aeronáutica en Honduras; específicamente la población entrevistada se puede dividir en cuatro grupos, 1) profesionales que laboran en instituciones dedicadas a la educación aeronáutica, 2) profesionales de la Agencia Hondureña de Aeronáutica Civil (AHAC), antes DGAC que laboran en la auditoría aeronáutica, 3) profesionales que han emprendido pequeñas o medianas empresas y, 4) profesionales que laboran en empresas de servicios aeronáuticos.

\section{Resultados}

Los resultados principales de la investigación son tres, el principal es la definición de un perfil profesional y técnico aeronáutico elaborado según el esquema sugerido por el Modelo Educativo de la UNAH; esta propuesta encuentra su fundamentación empírica en dos resultados más que ofrecen, el primero, una visión regional e internacional del mercado en el cual sería empleado el profesional egresado de una carrera aeronáutica universitaria, el segundo, un registro de las características profesionales y técnicas que se privilegian en las empresas del rubro aeronáutico en Honduras.

\section{- Datos del ámbito internacional}

La industria de las aerolíneas se encuentra en un proceso de recuperación de la disminución del tráfico debida a la crisis financiera y económica global de 2008, al mismo tiempo que se hacen ajustes para la reimplementación de su capacidad, pero ya el proceso de crecimiento ha retomado su camino. Por ejemplo, para el año 2010, el total de aeronaves comerciales se estimaba en 61,833, pronosticándose que para el año 2030 será casi tres veces más, con 151,565 distribuidas por todo el mundo (OACl, 2011). 


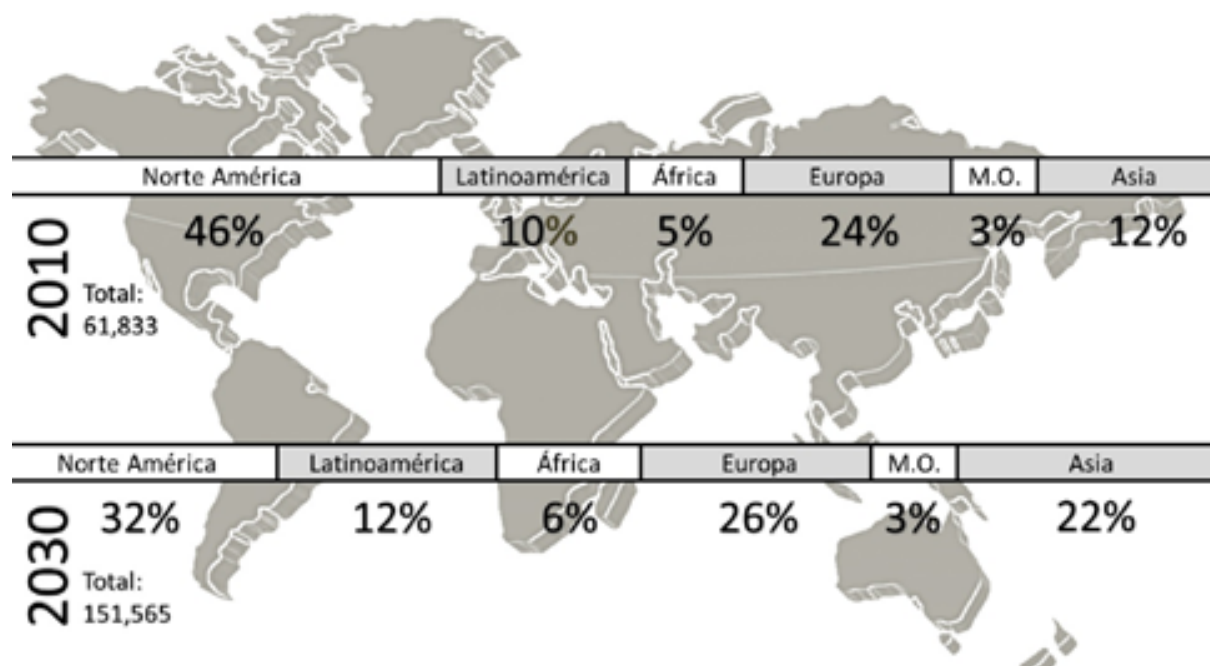

Gráfico 1: Distribución geográfica de aeronaves comerciales y su pronóstico a 20 años (Fuente OACI 2011)

Este aumento en el número de aeronaves provocará incremento del porcentaje de aeronaves en la misma etapa de tiempo, como lo muestra la gráfica: Latinoamérica pasará de un 10 a un 12\%, África se moverá de un 5 a un 6\%, Europa de un 24 a un $26 \%$ y Asia experimentará el crecimiento mayor pasando de un $12 \mathrm{a}$ un $22 \%$.

En lo que respecta al tráfico aéreo programado, los datos revelan que en el promedio de crecimiento anual, Latinoamérica se ubica en segundo lugar en el mundo, superada solamente por Asia y doblando el crecimiento que se proyecta para el mismo período para Norte América.

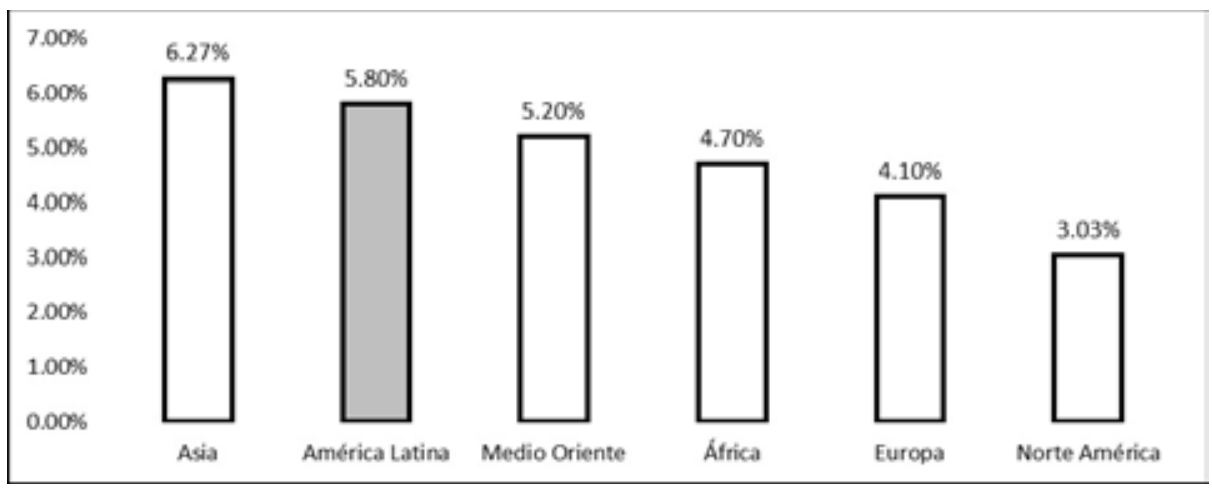

Gráfico 2: Promedio de Crecimiento Anual del Tráfico Aéreo (Fuente: OACI 2011) 
Es de esperarse que esta tendencia de crecimiento en los pronósticos de flotas y tráfico aéreo genere una necesidad de la dotación de personal, específicamente de pilotos, personal de mantenimiento de aeronaves y controladores de tránsito aéreo así como de personal de apoyo a las operaciones. Esta identificación geográfica no debe hacer olvidar que una de las características de la industria es su condición verdaderamente transnacional (Organización Internacional del Trabajo, 2013) rasgo que se corresponde con su reglamentación y las áreas geográficas de cobertura de sus empresas.

Pero el desafío está en tener una capacidad instalada para la capacitación de esta nueva población, capacidad que se ve mermada por la poca cantidad de centros de estudios que ostentan estas propuestas educativas.

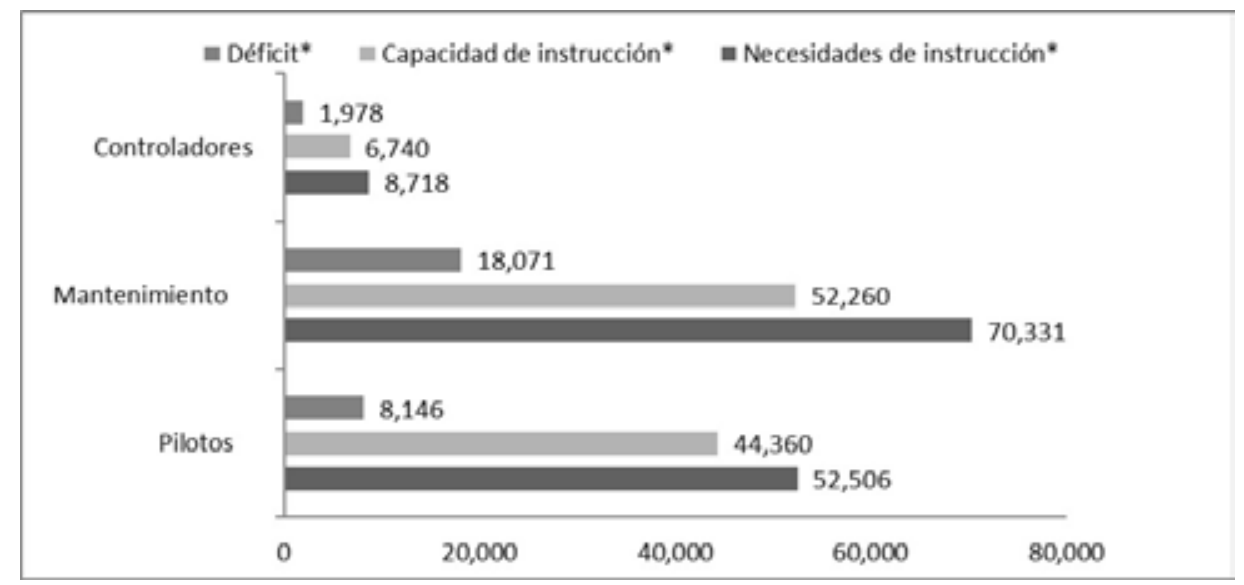

Gráfico 3: Cálculo estimado anual promedio comparativo entre la capacidad de instrucción y la necesidad de instrucción en el Mundo (OACl 2011)

Los datos reflejan déficits para los tres perfiles estudiados, lo cual representa un imperativo para que los centros de formación se preocupen más por la infraestructura, equipamiento y capacitación del propio personal. De no tomar medidas para afrontarlos, estos déficits se podrían convertir en una dificultad que reduciría el crecimiento del sector. Los esfuerzos por cubrir la demanda de cobertura no deben hacerse en detrimento de la calidad de los egresados de los procesos formativos, de ahí que deba prestarse atención de forma paralela a los cambios en los requerimientos de perfiles que demanda la industria. 
Latinoamérica, como es de esperarse sigue la tendencia mundial en dos de los tres perfiles de profesional mencionados. Sería de esperarse que los centros de formación, las empresas del rubro y las Autoridades Aeronáuticas de la región lean estos déficits como una oportunidad para emprender iniciativas que definan, revisen y actualicen los perfiles de egreso en diálogo con los empresarios latinoamericanos y de otras latitudes sin olvidar por otra parte las necesidades propias de las sociedades de la región. Asumir esta apertura tendría dos efectos positivos, por una parte respondería al mundo globalizado de la industria aeronáutica y por otra validaría los conocimientos comunes utilizados por la Academia en distintas regiones para la resolución de problemas del rubro (Beneitone, Esquetini, González, Maletá, \& Siufi, 2008).

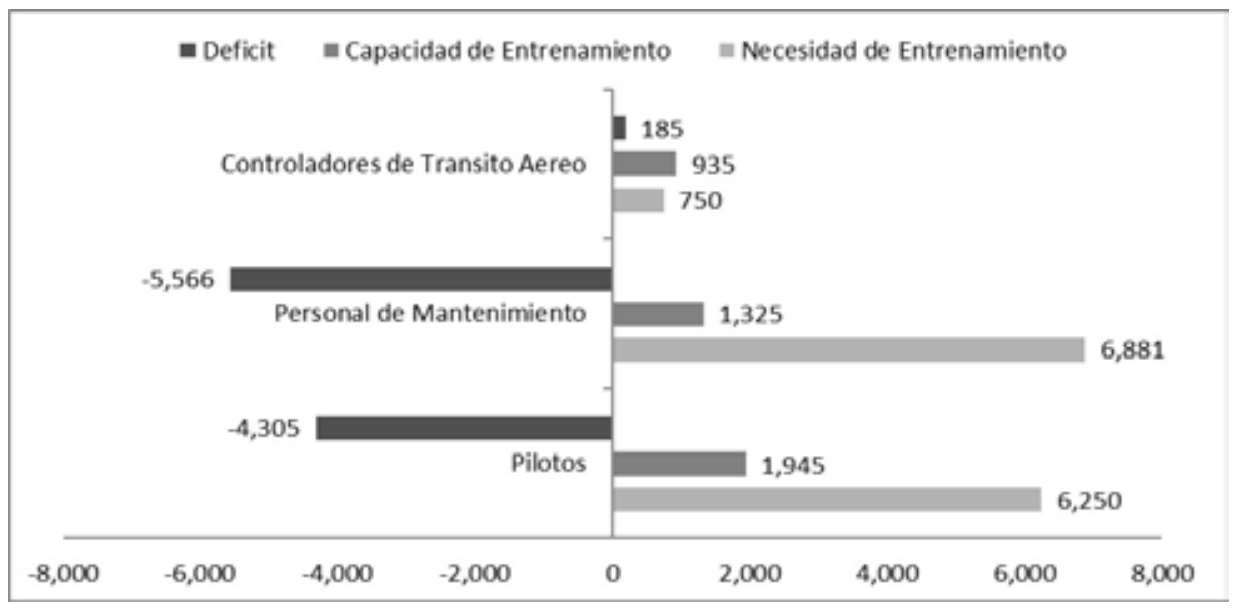

Gráfico 4: Capacidad de los Centros de Formación por número de estudiantes a atender en Latinoamérica (OACI 2011)

Con la exclusión de los controladores de tránsito aéreo, quienes disfrutan de un superávit, las especialidades de pilotos y personal de mantenimiento muestran déficits en su capacidad instalada de centros de entrenamiento, lo que se avizora repercutirá grandemente en la falta de personal especializado en la región.

\section{- Entrevista a representantes de empresas aeronáuticas}

La consulta fue dirigida a profesionales en niveles gerenciales de empresas o instituciones cuyas principales características son la posesión de grado universitario (licenciatura o ingeniería), experiencia de al menos cinco años en el campo de la aeronáutica, vinculación profesional actual con el campo y la capacidad de toma de decisiones para la contratación de personal. Esto marca un punto de partida 
importante considerando que los entrevistados son conocedores de la industria aeronáutica y están interactuando con ella, con lo cual conocen de sobra la problemática de la aeronáutica y seguramente han propuesto alternativas las cuales ahora estarían anuentes a proponer desde una perspectiva civil y académica.

Para empezar el $100 \%$ de las organizaciones aeronáuticas consultadas establecen que el título de educación superior es determinante al momento de la contratación. Estableciendo de esta manera lo que el sector contratante requiere. Considerando que el nivel de educación superior ofrece en la actualidad varias opciones de nivel de formación, es necesario definir la figura educativa a nivel superior que deberá ser diseñada; ante una pregunta que ofrecía varias opciones de respuesta, la opción más votada, como se muestra en el gráfico, fue la de Licenciatura.

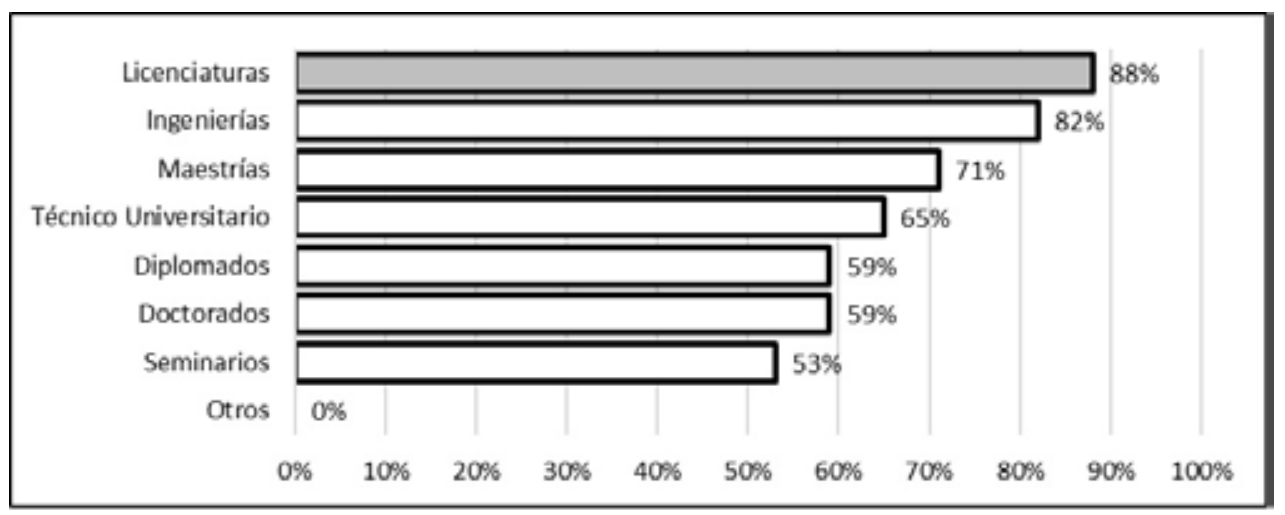

Gráfico 5: Preferencia de Tipo de Educación Aeronáutica del Nivel Superior para empresas aéreas (Fuente: entrevista aplicada).

Se puede decir que en este caso los entrevistados estarían buscando el complemento necesario de una industria que a pesar de contar con un alto nivel de complejidad y requerir de la utilización de equipos, sistemas y software de última generación, está conformada por personal técnico que muchas veces cuenta no con el grado universitario o bien ha recibido formación en la educación superior en campos distintos a los propios aeronáuticos (Colindres \& Matamoros, 2014).

En la interrogante planteada sobre los conocimientos procedentes de la formación se pidió a los entrevistados que valoraran de 1 a 4 una serie de conocimientos según la importancia que le dan a éstos en el ámbito empresarial aeronáutico. 


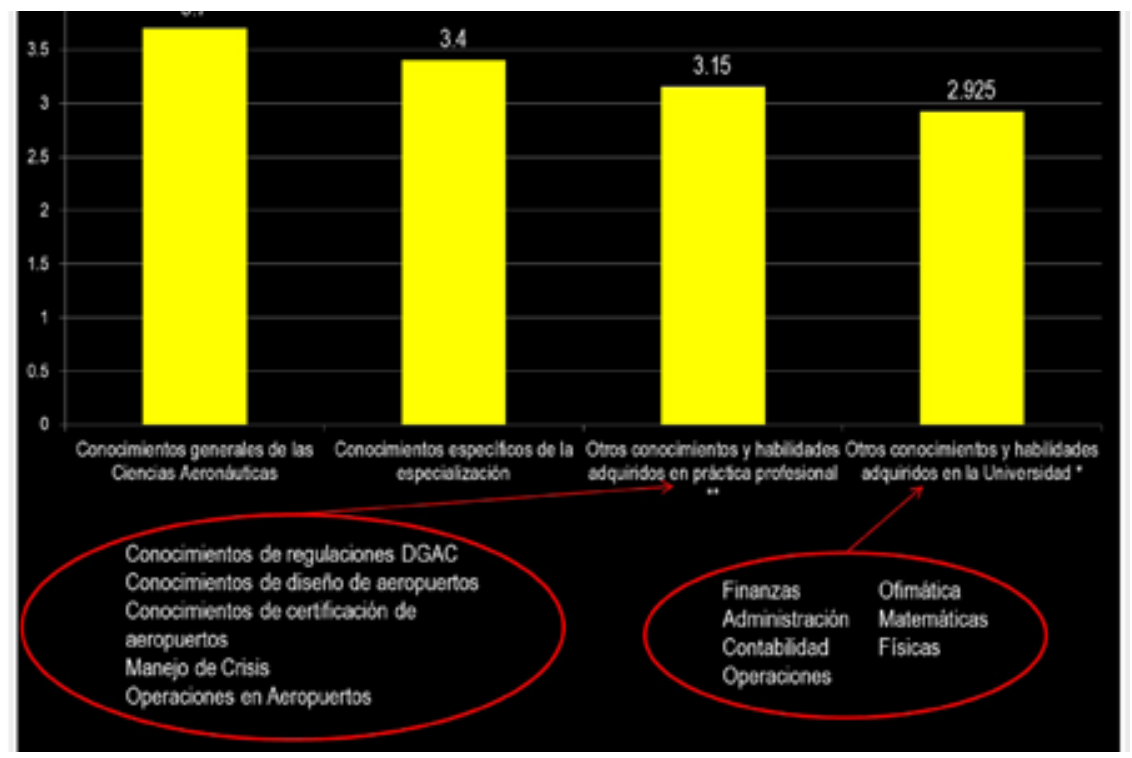

Gráfico 6: Valoración de los conocimientos procedentes de la formación al momento de una contratación por una entidad aeronáutica (Fuente: entrevista aplicada).

Esta gráfica muestra cómo la mayoría de las empresas consideran muy importante el conocimiento general de las Ciencias Aeronáuticas así como los conocimientos específicos de la especialización. Esta opción de los profesionales entrevistados se corresponde con el hecho de que en este campo se cuente en muchos casos con personal que cuenta con formación universitaria en otros campos, pero que cuentan con muchos conocimientos que pueden ser aplicados en el área aeronáutica, se trata por ejemplo de Ingenieros Civiles, Mecánicos, Industriales o Mecatrónicos; Licenciados en Administración de empresas, Mercadotecnia, Negocios Internacionales, arquitectos u otros perfiles similares. 


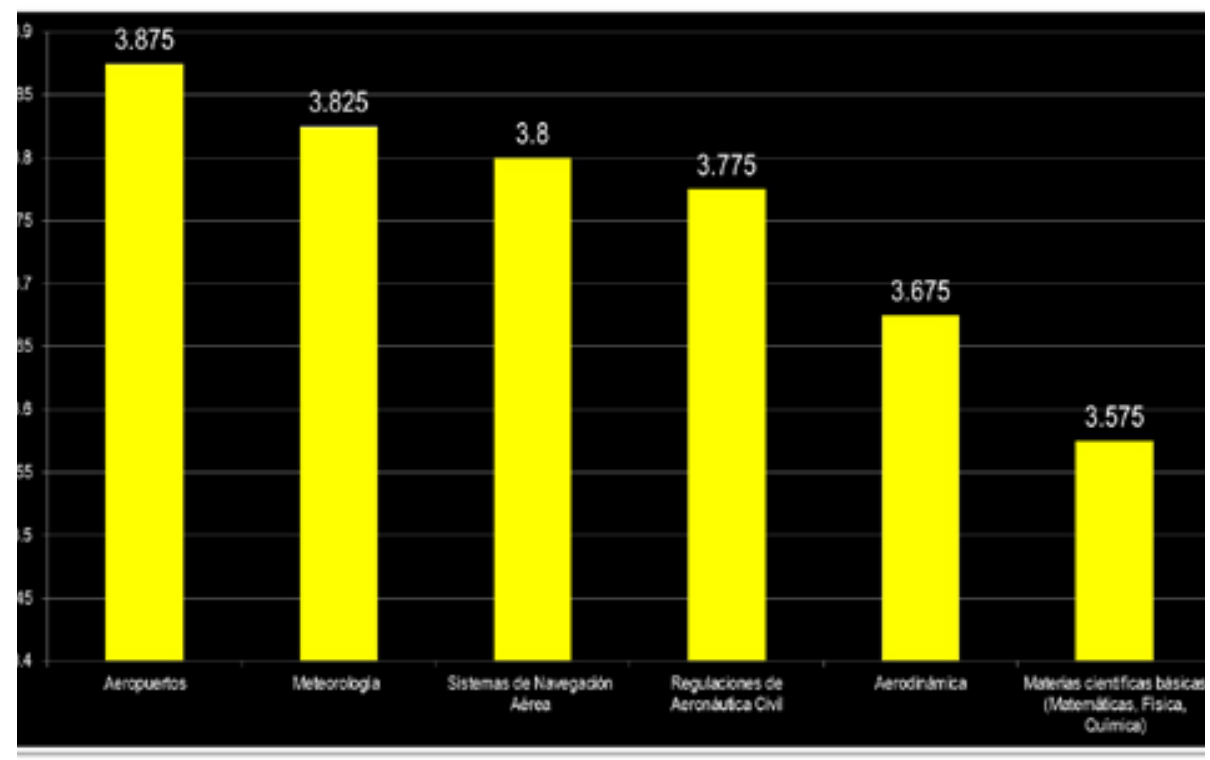

Gráfico 7: Valoración de los conocimientos generales del ambiente aeronáutico al momento de una contratación por una entidad aeronáutica (Fuente: entrevista aplicada)

En relación a la valoración de los conocimientos generales del ambiente aeronáutico, en la figura anterior se muestra que los más valorados de estos conocimientos son los relativos a Aeropuertos siendo los menos valorados la Aerodinámica y las Materias Científicas Básicas.

Los sistemas de Navegación Aérea y las Regulaciones Aeronáuticas están muy cercanas en su puntuación lo que muestra una relación ineludible entre ellas, quedando situadas, en importancia, en un punto intermedio dentro del conjunto.

La pregunta en la que se valoraron los conocimientos específicos de la especialización aeronáutica buscaba evaluar el requisito al momento de contratar profesionales con título universitario en Ciencias Aeronáuticas o en otros campos similares. Es muy probable que este perfil profesional vaya a ocupar puestos de nivel gerencial en la conducción de procesos, elaboración como responsable directo de informes y toma de decisiones relevantes dentro de la empresa o institución, lo cual le da una importancia considerable. 


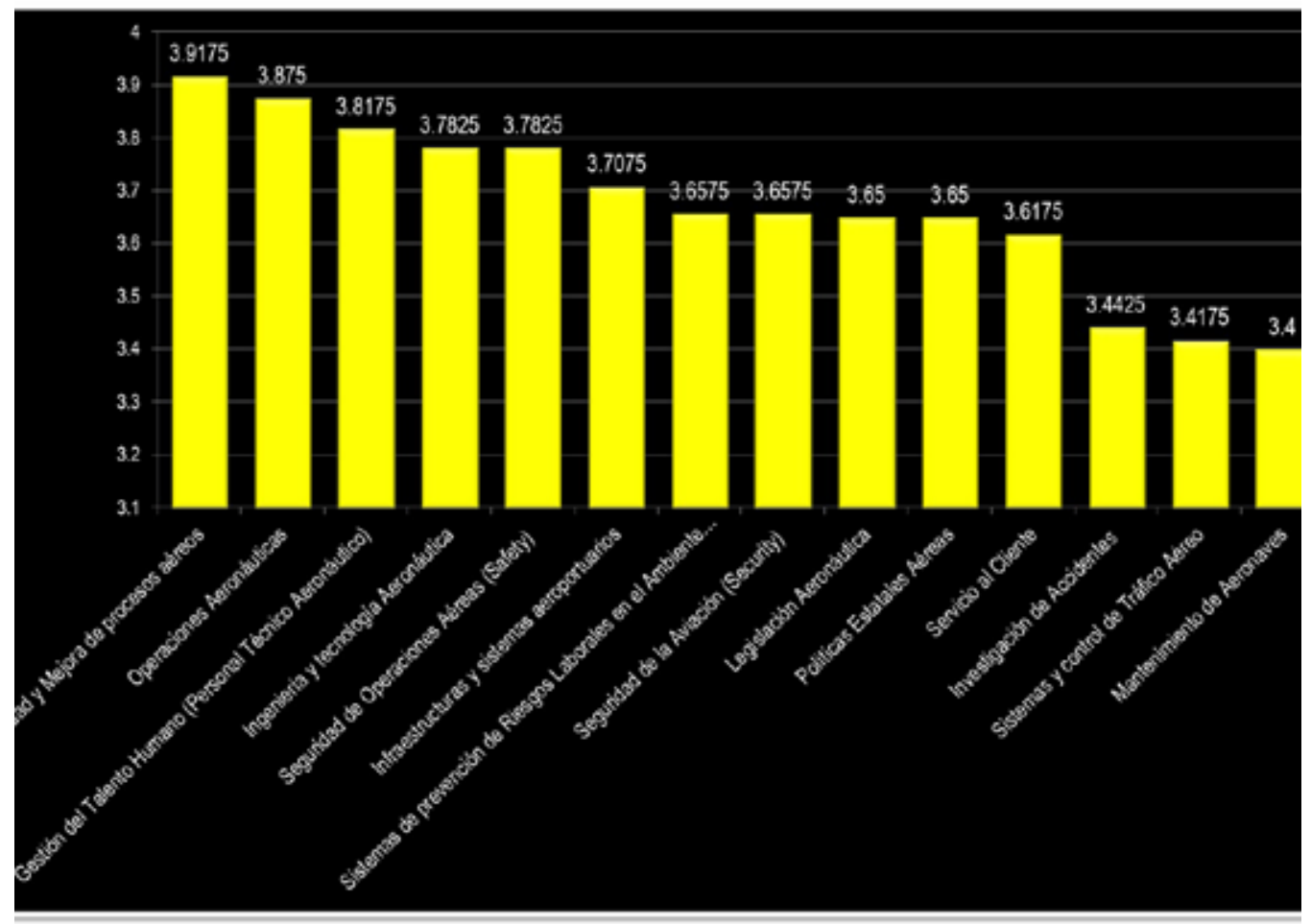

Gráfico 8: Valoración de los conocimientos específicos de la especialización al momento de una contratación por una entidad aeronáutica (Fuente: entrevista aplicada).

Dentro de los conocimientos de la especialización, los más valorados son la Calidad y Mejora de los procesos aéreos y las Operaciones Aeronáuticas; por otro lado, lo menos valorados, corresponden a las áreas de Investigación de Accidentes, Sistemas y Control de Tráfico Aéreo y Mantenimiento de Aeronaves. Se considera que los entrevistados al responder a esta pregunta se han decantado por así decirlo, a la parte más visible de la aeronáutica, relegando a los últimos lugares ámbitos de trabajo que propenden más a la investigación (investigación de accidentes aéreos) o que son menos visibles a los ojos de los usuarios de los servicios aeronáuticos (control de tránsito aéreo y mantenimiento de aeronaves).

Una de las interrogantes relevantes en la entrevista, fue la orientada a la valoración de capacidades de los prospectos a contratar; esta vez se pidió valorar en una escala de 1 a 4 una lista de capacidades, en dónde los valores más cercano al 1 serían los peor calificados y los más lejanos al 1 los mejor calificados. 


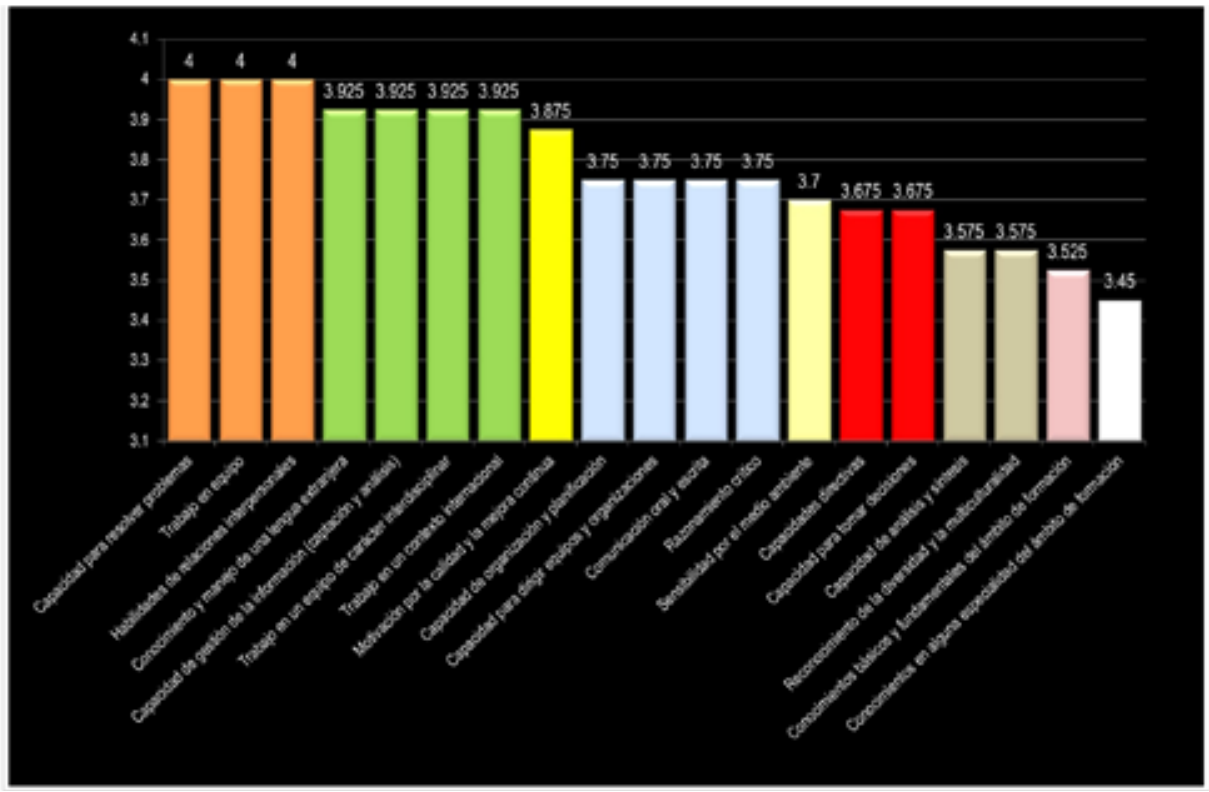

Gráfico 9: Valoración de las competencias profesionales al momento de una contratación por una entidad aeronáutica (Fuente: entrevista aplicada)

El rango de medias está entre 3.45 y 4.0 lo cual refleja unos resultados bastante alineados entre las competencias a las que se asigna más importancia (capacidad para resolver problemas, trabajo en equipo, habilidades de relaciones interpersonales) y aquella a la que se le asigna menos importancia (conocimiento en alguna especialidad del ámbito de formación). Con estos resultados específicos se puede demostrar la dificultad que puede experimentar un contratante al momento de descartar habilidades, puesto que lo más probables es que quisiera que los contratados tuvieran todas las habilidades. Pero por otra parte se sigue privilegiando el tipo de destrezas que ayudan más al día a día de la empresa que a la investigación para la innovación.

\section{- Perfiles de profesionales y técnicos universitarios}

Tomando en consideración la demanda internacional de profesionales y técnicos aeronáuticos a nivel internacional y las preferencias de niveles gerenciales de la industria y la institucionalidad aeronáutica hondureña, se considera oportuno hacer una propuesta desde el nivel de educación superior que ofrezca dos opciones de formación, la de una Licenciatura en Operaciones Aeronáuticas o bien la de un Técnico en Operaciones Aeronáuticas; en ambos casos se incluye el desglose 
de los conocimientos, competencias y valores necesarios para llevar a la práctica el perfil descrito. En todo momento se hace eco del modelo educativo de la UNAH puesto que se toma en consideración las características especiales del estudiantado al que se acogerá para formarlos como profesionales integrales que incorporen: conocimientos, procedimientos, hábitos, usos y costumbres, competencias, valores y habilidades personales (UNAH, 2009, p.26).

\section{Perfil Licenciado en Operaciones Aeronáuticas}

El Licenciado en Operaciones Aeronáuticas es un profesional universitario que ha desarrollado conocimientos y destrezas que le permiten continuar estudios de postgrado en campos afines o complementarios. Es un profesional abierto a formar parte de equipos de trabajo de investigación con egresados de carreras de cualquiera de las Escuelas y Departamentos de la Facultad de Ciencias Espaciales o de otras Facultades de la UNAH. Podrá trabajar como administrador de aeródromos y empresas de servicios aéreos, como funcionario de la autoridad aeronáutica nacional, como instructor de personal técnico o administrativo empleado en el área aeronáutica en general u otros campos similares en el ambiente público o privado; igualmente dependiendo de la especialidad técnica aeronáutica que decida cursar, podrá desempeñarse de acuerdo a su licencia aeronáutica acreditada por la Agencia Hondureña de Aeronáutica Civil.

Conocimientos:

- En materias generales: Español, Historia de Honduras, Sociología, Cultura Física y Deportes, Filosofía, Administración.

- Conocimientos básicos en el área de Físico-Matemático, con aplicación al ambiente aeronáutico.

- Conocimientos de la administración y gestión de recursos en donde se conjugan la administración de recursos humanos, la micro y macroeconomía, la legislación empresarial y laboral, la contabilidad, el mercadeo de productos y servicios, el comercio internacional, las finanzas y preparación, evaluación y administración de proyectos de inversión.

- Los conocimientos necesarios para un adecuado control, gestión e innovación empresarial.

- Conocimientos propios del profesional técnico aeronáutico establecido por la Agencia Hondureña de Aviación Civil a través de las regulaciones aéreas ne- 
cesarias para la acreditación técnica para la operación de equipo aeronáutico 0 para laborar en el área de operaciones de una empresa aeronáutica certificada.

- En conocimientos propiamente aeronáuticos para la gestión, regulación y control de un aeródromo o empresa aeronáutica pudiendo certificarla 0 administrarla y hacerla económicamente rentable, respetando la calidad del servicio al cliente y que simultáneamente sea amigable con el medio ambiente.

Competencias:

- Manejar responsablemente una empresa aeronáutica o aeroportuaria, o participar de agrupaciones de consultoría aeronáutica.

- Desempeñar eficientemente y con seguridad las funciones correspondientes a un técnico aeronáutico en cualquiera de las modalidades que ofrecidas en el Departamento de Ciencias Aeronáuticas.

- Organizar y dirigir grandes y pequeñas empresas aeronáuticas, con una visión internacional.

- Comprensión de lectura. Entender oraciones y párrafos escritos en documentos relacionados técnicos y administrativos aeronáuticos.

- Ciencia. Uso de normas y métodos científicos para proponer, investigar y resolver problemas dentro del campo aeronáutico.

- Planificar y ordenar los diversos aspectos organizacionales de una empresa o institución aeronáutica, a fin de anticipar y neutralizar situaciones negativas 0 conflictivas que puedan perjudicar la actividad cotidiana de producción, venta y negociación.

- Aprendizaje activo. Entender las implicaciones de la nueva información para la actual y futura resolución de problemas y toma de decisiones.

- Pensamiento Crítico. Usar la lógica y el razonamiento para identificar las fortalezas y debilidades de soluciones alternativas, conclusiones o enfoques a los problemas.

- Escritura. Comunicarse eficazmente por escrito y de acuerdo a las necesidades de la audiencia. 
- Matemáticas. Hacer aplicaciones prácticas de principios matemáticos para resolver problemas del mundo laboral y en las formaciones impartidas dentro de los equipos de trabajo de los que forme parte.

- Comunicación oral. Establecer comunicación oral con otros para transmitir información propia del campo de manera eficaz.

- Interactuar con sistemas computarizados, haciendo uso de equipos y sistemas informáticos ya sea administrativos o de control de un equipo automatizado aeronáutico 0 de posicionamiento global de manera real 0 a través de simuladores.

- Análisis de datos. Identificación de los principios subyacentes, las razones o los hechos de la información, descomponiendo la información o datos en partes separadas.

Valores:

- Luchar por el cumplimiento cabal de las leyes y regulaciones aeronáuticas para erogar cualquier actividad ilegal que vaya en contra de la seguridad en las operaciones aéreas.

- Promover el humanismo pleno, capaz de concebir al hombre en su exacta dimensión con cuerpo y con espíritu, con individualidad y colectividad.

- Comprometerse con su superación, mediante una continua actualización de sus conocimientos.

- Buscar siempre el éxito con responsabilidad y honestidad en el desempeño de sus labores.

- Promover y fomentar el patriotismo e interés en el servicio y participación comunitaria.

- Cultivar el espíritu emprendedor bajo estándares de calidad y productividad.

- Valorar y construir cualidades del liderazgo positivo para el desarrollo sostenible.

- Ser tolerante y amante de la cultura de paz. 
- Tener espíritu de innovación.

- Ser una persona honesta con ética profesional y alto sentido de responsabilidad.

- Amar la vida en todas sus manifestaciones.

\section{Perfil del Técnico Universitario en Operaciones Aeronáuticas}

El graduado como Técnico Universitario en Operaciones Aeronáuticas, es un profesional universitario con conocimientos que le permiten desempeñarse en sus labores en el campo aeronáutico aplicando conocimientos impartidos con los estándares que aplica la Educación en su Tercer Nivel en Honduras. Este profesional está en grado de formar parte de equipos de análisis y de generación de propuestas en empresas aéreas o instituciones del Estado vinculadas con la aeronáutica. Cuando su acreditación así lo indique, podrá trabajar como Piloto Comercial e Instructor en el área de operaciones de vuelo en la aviación comercial, como Despachador de Vuelo en una aerolínea u otra empresa similar o como Controlador de Tránsito Aéreo en institución del Estado.

Conocimientos:

- En materias generales: Español, Historia de Honduras, Sociología, Cultura Física y Deportes, Filosofía, Administración.

- Conocimientos básicos en el área de Físico-Matemático, con aplicación al ambiente aeronáutico.

- Conocimientos propios del profesional técnico aeronáutico establecido por la Agencia Hondureña de Aeronáutica Civil, a través de las regulaciones aéreas necesarias para la acreditación técnica para la operación de equipo aeronáutico o laborar en el área de operaciones de una empresa aeronáutica certificada.

Competencias

- Participar en agrupaciones de consultoría aeronáutica, haciendo aportes propios de un Técnico Universitario.

- Desempeñar eficientemente y con seguridad las funciones técnicas aeronáuticas aplicando los conocimientos propios del nivel universitario. 
- Entender oraciones y párrafos escritos en documentos relacionados técnicos y administrativos aeronáuticos en el lenguaje propio del campo.

- Colaborar en grupos de investigación y resolución de problemas dentro del campo aeronáutico.

- Capacidad para hacer aportes significativos en procesos de planificación y organización de una empresa o institución aeronáutica, a fin de anticipar y neutralizar situaciones negativas o conflictivas que puedan afectar negativamente las operaciones de vuelo.

- Entender las implicaciones de la nueva información para la actual y futura resolución de problemas y toma de decisiones.

- Usar la lógica y el razonamiento para identificar las fortalezas y debilidades de soluciones alternativas, conclusiones o enfoques a los problemas.

- Comunicarse de manera eficaz oralmente y por escrito de acuerdo a las necesidades de la audiencia.

- Hacer aplicaciones prácticas de principios matemáticos para resolver problemas del mundo laboral y en las formaciones impartidas dentro de los equipos de trabajo de los que forme parte.

- Interactuar con sistemas computarizados, haciendo uso de equipos y sistemas informáticos ya sea administrativos o de control de un equipo automatizado aeronáutico 0 de posicionamiento global de manera real o a través de simuladores.

Valores:

- Luchar por el cumplimiento cabal de las leyes y regulaciones aeronáuticas para erogar cualquier actividad ilegal que vaya en contra de la seguridad en las operaciones aéreas.

- Promover el humanismo pleno, capaz de concebir al hombre en su exacta dimensión con cuerpo y con espíritu, con individualidad y colectividad.

- Comprometerse con su superación, mediante una continua actualización de sus conocimientos.

- Buscar siempre el éxito con responsabilidad y honestidad en el desempeño de sus labores. 
- Promover y fomentar el patriotismo e interés en el servicio y participación comunitaria.

- Valorar y construir cualidades del liderazgo positivo para el desarrollo sostenible.

- Ser tolerante y amante de la cultura de paz.

- Tener espíritu de innovación.

- Ser una persona honesta con ética profesional y alto sentido de responsabilidad.

\section{Conclusiones y recomendaciones}

\section{Conclusiones}

- El abordaje de los contenidos formativos en aeronáutica requiere en primer instancia del apoyo específico de los campos que integran la industria; a este primer grupo se deben sumar otros actores vinculados con el campo, entre los que se pueden mencionar las escuelas de vuelo, la institucionalidad del Estado que regula la aeronáutica civil y los profesionales independientes aeronáuticos y no aeronáuticos relacionados con este campo por vínculos diversos.

- Uno de los principales aportes que puede hacer la UNAH en el campo aeronáutico es darle cohesión a la labor hasta ahora dispersa de los distintos actores vinculados con el tema; esta labor puede darse posicionando una agenda de temas de País relacionados con el campo aeronáutico. La Licenciatura en Operaciones Aeronáuticas es un buen primer paso para la construcción y puesta en práctica de esta agenda.

- De acuerdo con la demanda de formación percibida, los perfiles profesionales encontrados y la infraestructura instalada de la industria aeronáuticas, en una carrera de grado universitario, se podrían tener varias salidas relacionadas con distintos técnicos, desde ahora se pueden ofrecer los técnicos para piloto de ala fija, para piloto de ala rotatoria, para despachador de vuelo y para control de tránsito aéreo; con un componente consolidado en Administración de Aeropuertos cuando se opte por la Licenciatura.

- La orientación hacia la administración de aeropuertos de la Licenciatura en Operaciones Aeronáuticas hace que los contenidos y las metodologías peda- 
gógicas a utilizar en el proceso formativo requieran de la colaboración de otros Departamentos de la UNAH.

- Honduras tiene una tradición aeronáutica con un potencial significativo de nuevos crecimientos en distintas direcciones; estos nuevos desarrollos deben ajustarse a las corrientes globales actuales para fortalecernos como País con la competitividad necesaria para responder a una demanda internacional con altas exigencias.

- Es una prioridad de primer orden para Honduras el desarrollo de la ciencia y la tecnología aeronáuticas en una perspectiva civil que tome en cuenta las dimensiones globales del crecimiento de la industria y la demanda creciente de movilización de personas a nivel mundial.

- Es de importancia estratégica inventariar los recursos y las capacidades con los que se cuenta como País para dar respuesta a las necesidades presentes y futuras de personal e infraestructura aeronáutica; este ejercicio analítico y prospectivo se puede hacer en tres perspectivas: la planificación de recursos humanos técnicos y administrativos, el desarrollo de capacidad institucional para la regulación y el impulso de la aeronáutica civil y la definición de necesidades de financiamiento y establecimiento de políticas públicas para el campo.

\section{Recomendaciones}

- El desarrollo de una oferta académica para la aeronáutica civil en Honduras debe tener como referente principal el sistema aeroportuario del País, propiciando un diálogo amplio y sistemático con los actores que interactúan en este ámbito.

- Los aeródromos deben ser potenciados como elementos estratégicos en la Visión de País y Plan de Nación para dinamizar la movilidad interna en un País en donde persisten las dificultades que supone una geografía accidentada. Este rescate de los aeródromos debería estar orientado a coadyuvar a un mejor posicionamiento de los profesionales aeronáuticos egresados.

- El lanzamiento de una Licenciatura en campo aeronáutico no puede descuidar los esfuerzos para el establecimiento de otras ofertas académicas de pregrado y grado; en tal sentido de forma simultánea al funcionamiento de la Licenciatura se debe fortalecer la oferta de educación no formal ya existente con nuevos diplomados, seminarios, talleres, ciclos de conferencias, entre otros y, se debe 
entrar en la etapa de conformación de un nuevo grado que podría tener una orientación hacia la ingeniería aeronáutica.

- Puesto que el Departamento de Ciencias Aeronáuticas cuenta ya con un banco de datos de profesionales aeronáuticos hondureños y varios contactos internacionales que han mostrado anuencia a colaborar, se debe mantener la relación con los primeros y sancionar mediante convenios generales y específicos la relación con los segundos, al momento de entrar en la fase de funcionamiento de la Licenciatura en Operaciones Aeronáuticas.

- Dada la naturaleza regional e internacional de las ciencias y de la industria aeronáutica, una propuesta de posgrado en este campo debe asumir una cobertura regional. Para emprender este esfuerzo con garantías de éxito se debe estrechar vínculos de colaboración con instituciones académicas y organismos internacionales que se sabe, están interesados en la aeronáutica en Centro América.

\section{Bibliografía}

- Beneitone, P., Esquetini, C., González, J., Maletá, M., \& Siufi, G. W. (2008). Refleiones y perspectivas de la edución superior en América Latina. Informe Final, Proyecto Tuning, América Latina 2004-2007.

- Colindres, A., \& Matamoros, A. (2014). Cuerpo de contenidos para la Formación de Controlador de Tránsito Aéreo el Ambito de la Ciencias Aeronáuticas en Honduras. Tegucigalpa: No publicado.

- María Cristina Pineda, O. A. (2012). Bases para el establecimiento de las Ciencias Aeroáuticas en la Facultad de Ciencias Aeronáuticas en la UNAH. Tegucigalpa: Editorial Universitaria.

- OACI. (2011). Global and Regional 20-Year Forecasts. Montreal: OACI.

- Organización Internacional del Trabajo. (2013). La aviación civil y los cambios en su entorno laboral. Ginebra: OIT.

- UNAH. (2009). El modelo educativo de la UNAH. Tegucigalpa: Editorial Universitaria. 